\title{
A new kind of representations on noncommutative phase space
}

\author{
Sicong Jing*, Bingsheng Lin \\ Department of Modern Physics, University of Science and Technology of China \\ Hefei, Anhui 230026, China
}

20 May 2008

\begin{abstract}
We introduce new representations to formulate quantum mechanics on noncommutative phase space, in which both coordinate-coordinate and momentum-momentum are noncommutative. These representations explicitly display entanglement properties between degrees of freedom of different coordinate and momentum components. To show their potential applications, we derive explicit expressions of Wigner function and Wigner operator in the new representations, as well as solve exactly a two-dimensional harmonic oscillator on the noncommutative phase plane with both kinetic coupling and elastic coupling.
\end{abstract}

PACS: 03.65.-w; 03.65.Fd; 03.65.Ud; 02.40.Gh

Keywords: Noncommutative phase space; Entangled state; Wigner function; Coupled harmonic oscillator

\section{Introduction}

As is well known, representations and transformation theories, founded by Dirac [1, play basic and important role in quantum mechanics. Many quantum mechanics problems were solved cleverly by working in specific representations. Some representations, such as, the coordinate, the momentum, the number representation, as well as the coherent state representation, are often employed in the literature of ordinary quantum mechanics. In noncommutative quantum mechanics (NCQM) [2]-4], because of the noncommutativity of coordinate-component (and/or momentum-component) operators, there are no simultaneous eigenstates for these different coordinate (or momentum) operators, and one can hardly construct a coordinate (or momentum) representation in the usual sense. However, in order to formulate quantum mechanics on a noncommutative phase space so that some dynamic problems can be solved, we do need some appropriate representations. Here some few words on the noncommutativity of momentum-component operators are useful. Although in string theory only the coordinate space exhibits a noncommutative structure, some authors have studied models in which a noncommutative geometry is defined on the whole phase space [5, 6]. Noncommutativity between momenta arises naturally as a consequence of noncommutativity between coordinates, as momenta are defined to be the partial derivatives of the action with respect to the noncommutative coordinates [7].

\footnotetext{
${ }^{*}$ Corresponding author.

${ }^{1}$ E-mail addresses: sjing@ustc.edu.cn (S. Jing), xylbs@ustc.edu (B. Lin).
} 
On the other hand, the usual method to study NCQM is using the Seiberg-Witten map to change a problem of NCQM into a corresponding problem of quantum mechanics on the commutative space. In the case of only the coordinate space is noncommutative, this method is consistent with the Weyl-Moyal correspondence which amounts to replacing the usual product in noncommutative space by a star product in ordinary space. This method, however, does not always work, for example, when both coordinates and momenta are noncommutative, i.e., on a noncommutative phase space, although using the Seiberg-Witten map one can write down a Hamiltonian of NCQM in terms of the ordinary commutative coordinates and momenta operators, one has no way to get a well-defined Schrödinger equation which is consistent with the corresponding star product. Therefore, it is necessary to develop other new method to solve the quantum mechanical problems on noncommutative phase space.

Noticing that although two coordinate-component (and/or momentum-component) operators on the noncommutative phase space do not commute each other, the difference of the two coordinate operators indeed commute with the sum of the relevant two momentum operators, thus we can still employ Einstein-Podolsky-Rosen's (EPR) [8] idea to construct entangled states on the noncommutative phase space. It is easily to show that the entangled states with continuum variables are orthonormal and satisfy completeness relations, therefore they present new representations for NCQM. The first bipartite entangled state representation of continuum variables is constructed by H. Fan and J. R. Klauder 9 based on the idea of quantum entanglement initiated by EPR who used commutative property of two particles' relative coordinate and total momentum. In this Letter, for the noncommutative phase space, by this we mean that both the coordinate-coordinate and the momentum-momentum operators are noncommutative, we construct continuum entangled state representations and study their some basic properties including orthonormality and completeness. Besides, we derive explicit expressions of Wigner function (WF) and Wigner operator in the new representations. To show the potential applications of the entangled state representations in NCQM, we solve exactly the energy level and WFs of a two-dimensional harmonic oscillator on a noncommutative phase plane with both kinetic coupling and elastic coupling.

The work is arranged as follows: In section 2 we construct the entangled state representations for the noncommutative phase space from a set of deformed boson commutation relations. These entangled states are orthonormal and complete, so that arbitrary state can be expended as their linear combination. We also evaluate matrix elements of noncommutative coordinate and momentum operators in these representations. Section 3 is devoted to derive explicit expressions of the WFs and the Wigner operator in the new representation. To show the potential role of the new representation, we study a two-dimensional oscillator on the noncommutative phase space with both kinetic coupling and elastic coupling in section 4, and obtain its energy spectrum and WFs exactly. Some summary and comments are in the last section.

\section{Entangled state representations for NCQM}

Without loss of generality and for the sake of simplicity, we only discuss the four-dimensional noncommutative phase space, in which both coordinate-coordinate and momentum-momentum are noncommutative. Operators $\hat{x}, \hat{y}, \hat{p}_{x}$ and $\hat{p}_{y}$ satisfy the following commutation relations

$$
[\hat{x}, \hat{y}]=i \mu, \quad\left[\hat{p}_{x}, \hat{p}_{y}\right]=i \nu, \quad\left[\hat{x}, \hat{p}_{x}\right]=\left[\hat{y}, \hat{p}_{y}\right]=i \hbar,
$$


and other commutators of these operators are vanishing, where $\mu$ and $\nu$ are real nonzero parameters with dimension of $(\text { length })^{2}$ and (momentum $)^{2}$ respectively. Considering the following operators

$$
\hat{R}=\frac{\hat{x}-\hat{y}}{\sqrt{2}}, \quad \hat{P}=\frac{\hat{p}_{x}+\hat{p}_{y}}{\sqrt{2}}, \quad \hat{Q}=\frac{\hat{x}+\hat{y}}{\sqrt{2}}, \quad \hat{K}=\frac{\hat{p}_{x}-\hat{p}_{y}}{\sqrt{2}} .
$$

obviously one finds that $\hat{R}$ and $\hat{P}$ are commute each other, as well as $\hat{Q}$ and $\hat{K}$ are commute each other, respectively. Thus $\hat{R}$ and $\hat{P}$ have simultaneous eigenstates $|\lambda\rangle$, and $\hat{Q}$ and $\hat{K}$ have simultaneous eigenstates $|\xi\rangle$. Here $\lambda$ and $\xi$ may be complex numbers, $\left(\lambda=\lambda_{1}+i \lambda_{2}\right.$, and $\xi=\xi_{1}+i \xi_{2}$ ), and $\lambda_{1}, \lambda_{2}, \xi_{1}$ and $\xi_{2}$ are real numbers.

In order to get explicit expressions of the eigenstates $|\lambda\rangle$ and $|\xi\rangle$, we use the following quadrature decomposition

$$
\begin{array}{ll}
\hat{x}=\sqrt{\frac{\hbar}{2}} \sqrt[4]{\frac{\mu}{\nu}}\left(\hat{a}+\hat{a}^{\dagger}\right), & \hat{p}_{x}=\frac{1}{i} \sqrt{\frac{\hbar}{2}} \sqrt[4]{\frac{\nu}{\mu}}\left(\hat{a}-\hat{a}^{\dagger}\right), \\
\hat{y}=\sqrt{\frac{\hbar}{2}} \sqrt[4]{\frac{\mu}{\nu}}\left(\hat{b}+\hat{b}^{\dagger}\right), & \hat{p}_{y}=\frac{1}{i} \sqrt{\frac{\hbar}{2}} \sqrt[4]{\frac{\nu}{\mu}}\left(\hat{b}-\hat{b}^{\dagger}\right) .
\end{array}
$$

Obviously, these dimensionless operators $\hat{a}, \hat{b}$ and their Hermitian conjugates satisfy the following commutation relations

$$
\left[\hat{a}, \hat{a}^{\dagger}\right]=\left[\hat{b}, \hat{b}^{\dagger}\right]=1, \quad[\hat{a}, \hat{b}]=\left[\hat{a}^{\dagger}, \hat{b}^{\dagger}\right]=0, \quad\left[\hat{a}, \hat{b}^{\dagger}\right]=-\left[\hat{b}, \hat{a}^{\dagger}\right]=i \theta,
$$

where the dimensionless parameter $\theta=\frac{\sqrt{\mu \nu}}{\hbar}$. The algebraic relations in Eq. (44) are exactly same in form as the deformed boson algebra in [10]. To our knowledge, this type of deformed boson commutation relations also appeared in early work by Caves and Schumaker [11. Their quadrature-phase amplitudes satisfy the same commutation relations as Eq.(44). When $\theta=0$, the Eq.(4) reduces to ordinary boson algebra. It is worth pointing out that such a deformed boson algebraic relations derived in [10] were under an assumption of maintaining Bose-Einstein statistics (this assumption is equivalent to propose a direct proportionality between the noncommutative parameters $\mu$ and $\nu$ ). However, recently Bertolami and Rosa argued that there is no strong argument supporting such a direct proportionality relation [12. Here we obtain Eq.(44) from Eq.(10) without using any similar assumption, besides the quadrature decomposition (3).

In terms of these deformed boson operators, the operators $\hat{R}, \hat{P}, \hat{Q}$ and $\hat{K}$ can be expressed as

$$
\begin{array}{ll}
\hat{R}=\frac{\sqrt{\hbar}}{2} \sqrt[4]{\frac{\mu}{\nu}}\left(\hat{a}+\hat{a}^{\dagger}-\hat{b}-\hat{b}^{\dagger}\right), & \hat{P}=\frac{\sqrt{\hbar}}{2 i} \sqrt[4]{\frac{\nu}{\mu}}\left(\hat{a}-\hat{a}^{\dagger}+\hat{b}-\hat{b}^{\dagger}\right), \\
\hat{Q}=\frac{\sqrt{\hbar}}{2} \sqrt[4]{\frac{\mu}{\nu}}\left(\hat{a}+\hat{a}^{\dagger}+\hat{b}+\hat{b}^{\dagger}\right), & \hat{K}=\frac{\sqrt{\hbar}}{2 i} \sqrt[4]{\frac{\nu}{\mu}}\left(\hat{a}-\hat{a}^{\dagger}-\hat{b}+\hat{b}^{\dagger}\right) .
\end{array}
$$

The simultaneous eigenstate $|\lambda\rangle$ of $\hat{R}$ and $\hat{P}$ can be written as

$$
|\lambda\rangle=\exp \left(-\frac{|\lambda|^{2}}{2}+\theta \lambda_{1} \lambda_{2}+\lambda \hat{a}^{\dagger}-\lambda^{*} \hat{b}^{\dagger}+\frac{1}{1-\theta^{2}}\left(\hat{a}^{\dagger} \hat{b}^{\dagger}-\frac{i \theta}{2} \hat{a}^{\dagger 2}+\frac{i \theta}{2} \hat{b}^{\dagger 2}\right)\right)|00\rangle
$$


where $|00\rangle$ is a two-mode normalized boson vacuum state in the deformed Fock space satisfying $\hat{a}|00\rangle=0, \hat{b}|00\rangle=0$ and $\langle 00 \mid 00\rangle=1$. Sometimes $|\lambda\rangle$ is written as $\left|\lambda_{1}, \lambda_{2}\right\rangle$ to reflect its parameter dependence. Using the deformed boson commutation relation (4), one finds

$$
\left(\hat{a}-\hat{b}^{\dagger}\right)|\lambda\rangle=\left(\lambda-i \theta \lambda^{*}\right)|\lambda\rangle, \quad\left(\hat{b}-\hat{a}^{\dagger}\right)|\lambda\rangle=-\left(\lambda^{*}+i \theta \lambda\right)|\lambda\rangle,
$$

which lead to

$$
\hat{R}|\lambda\rangle=\sqrt{\hbar} \sqrt[4]{\frac{\mu}{\nu}}\left(\lambda_{1}-\theta \lambda_{2}\right)|\lambda\rangle, \quad \hat{P}|\lambda\rangle=\sqrt{\hbar} \sqrt[4]{\frac{\nu}{\mu}}\left(\lambda_{2}-\theta \lambda_{1}\right)|\lambda\rangle .
$$

Thus $|\lambda\rangle$ indeed is the simultaneous eigenstate of the operators $\hat{R}$ and $\hat{P}$.

Now we are at the position to consider the orthonormality of the $|\lambda\rangle$. From Eq.(17) one has

$$
\begin{aligned}
& \left\langle\lambda^{\prime}\left|\left(\hat{a}-\hat{b}^{\dagger}\right)\right| \lambda\right\rangle=\left(\lambda-i \theta \lambda^{*}\right)\left\langle\lambda^{\prime} \mid \lambda\right\rangle=\left(\lambda^{\prime}-i \theta \lambda^{\prime *}\right)\left\langle\lambda^{\prime} \mid \lambda\right\rangle, \\
& \left\langle\lambda^{\prime}\left|\left(\hat{b}-\hat{a}^{\dagger}\right)\right| \lambda\right\rangle=\left(-\lambda^{*}-i \theta \lambda\right)\left\langle\lambda^{\prime} \mid \lambda\right\rangle=\left(-\lambda^{\prime *}-i \theta \lambda^{\prime}\right)\left\langle\lambda^{\prime} \mid \lambda\right\rangle,
\end{aligned}
$$

or $\left(\lambda-\lambda^{\prime}-i \theta\left(\lambda^{*}-\lambda^{*}\right)\right)\left\langle\lambda^{\prime} \mid \lambda\right\rangle=0$ and $\left(\lambda^{*}-\lambda^{\prime *}+i \theta\left(\lambda-\lambda^{\prime}\right)\right)\left\langle\lambda^{\prime} \mid \lambda\right\rangle=0$, which imply that

$$
\left\langle\lambda^{\prime} \mid \lambda\right\rangle \propto \delta^{(2)}\left(\lambda-\lambda^{\prime}-i \theta\left(\lambda^{*}-\lambda^{*}\right)\right)=\delta^{(2)}\left(\lambda^{*}-\lambda^{\prime *}+i \theta\left(\lambda-\lambda^{\prime}\right)\right),
$$

where $\delta^{(2)}(z) \equiv \delta\left(z_{1}\right) \delta\left(z_{2}\right),\left(z=z_{1}+\mathrm{i} z_{2}\right)$. Without loss generality, one may regard the noncommutative parameter $\theta$ as a very small one. Eq.(10) means that only when $\lambda-\lambda^{\prime}=$ $i \theta\left(\lambda^{*}-\lambda^{\prime *}\right)$ as well as $\lambda^{*}-\lambda^{\prime *}=-i \theta\left(\lambda-\lambda^{\prime}\right)$ the inner product $\left\langle\lambda^{\prime} \mid \lambda\right\rangle$ has nonzero value. Substituting the latter into the former, one has $\lambda-\lambda^{\prime}=\theta^{2}\left(\lambda-\lambda^{\prime}\right)$, which means that only for the case of $\lambda-\lambda^{\prime}=0,\left\langle\lambda^{\prime} \mid \lambda\right\rangle$ does not vanish (please notice here $\theta^{2} \neq 1$ ). It is also true that only for the case of $\lambda^{*}-\lambda^{\prime *}=0,\left\langle\lambda^{\prime} \mid \lambda\right\rangle$ does not vanish. Therefore, we have $\left\langle\lambda^{\prime} \mid \lambda\right\rangle \propto \delta^{(2)}\left(\lambda-\lambda^{\prime}\right)$. In addition, for the later convenience, we take the proportional coefficient here as $\pi / \sqrt{1-\theta^{2}}$, so we have

$$
\left\langle\lambda^{\prime} \mid \lambda\right\rangle=\frac{\pi}{\sqrt{1-\theta^{2}}} \delta^{(2)}\left(\lambda-\lambda^{\prime}\right) .
$$

Then we show that $|\lambda\rangle$ satisfies the following complete relation

$$
\frac{\sqrt{1-\theta^{2}}}{\pi} \int d^{2} \lambda|\lambda\rangle\langle\lambda|=1
$$

where $d^{2} \lambda=d \lambda_{1} d \lambda_{2}$. In order to show it, we use an expression for the vacuum projection operator in the deformed Fock space

$$
|00\rangle\langle 00|=: \exp \left(-\frac{1}{1-\theta^{2}}\left(\hat{a}^{\dagger} \hat{a}+\hat{b}^{\dagger} \hat{b}-i \theta\left(\hat{a}^{\dagger} \hat{b}-\hat{b}^{\dagger} \hat{a}\right)\right)\right):,
$$

which will reduce to ordinary form when $\theta=0$, where the notation : ...: means take the normal ordering product for the operators $\hat{a}, \hat{b}$ and their Hermitian conjugate operators. Substituting Eq.(6) into Eq.(12), one has

$$
\begin{aligned}
& \frac{\sqrt{1-\theta^{2}}}{\pi} \int d \lambda_{1} d \lambda_{2}: \exp (\left.-\lambda_{1}^{2}-\lambda_{2}^{2}+2 \theta \lambda_{1} \lambda_{2}+\lambda \hat{a}^{\dagger}-\lambda^{*} \hat{b}^{\dagger}+\lambda^{*} \hat{a}-\lambda \hat{b}\right) \\
& \times \exp \left\{\frac{1}{1-\theta^{2}}\left(\hat{a}^{\dagger} \hat{b}^{\dagger}+\hat{a} \hat{b}-\frac{i \theta}{2}\left(\hat{a}^{\dagger 2}-\hat{b}^{\dagger 2}-\hat{a}^{2}+\hat{b}^{2}\right)\right)\right. \\
&\left.-\left(\hat{a}^{\dagger} \hat{a}+\hat{b}^{\dagger} \hat{b}-i \theta\left(\hat{a}^{\dagger} \hat{b}-\hat{b}^{\dagger} \hat{a}\right)\right)\right\}: .
\end{aligned}
$$


Now within the normal ordering product, one can treat the operators as $c$-numbers, so after integrating over $\lambda_{1}$ and $\lambda_{2}$, one gets the identity operator, which concludes the proof of Eq.(12).

Similarly, we may express the simultaneous eigenstate of $\hat{Q}$ and $\hat{K}$ as

$$
|\xi\rangle=\exp \left(-\frac{|\xi|^{2}}{2}-\theta \xi_{1} \xi_{2}+\xi \hat{a}^{\dagger}+\xi^{*} \hat{b}^{\dagger}-\frac{1}{1-\theta^{2}}\left(\hat{a}^{\dagger} \hat{b}^{\dagger}-\frac{i \theta}{2} \hat{a}^{\dagger 2}+\frac{i \theta}{2} \hat{b}^{\dagger 2}\right)\right)|00\rangle,
$$

which satisfies

$$
\hat{Q}|\xi\rangle=\sqrt{\hbar} \sqrt[4]{\frac{\mu}{\nu}}\left(\xi_{1}+\theta \xi_{2}\right)|\xi\rangle, \quad \hat{K}|\xi\rangle=\sqrt{\hbar} \sqrt[4]{\frac{\nu}{\mu}}\left(\xi_{2}+\theta \xi_{1}\right)|\xi\rangle .
$$

Also the $|\xi\rangle$ obeys the following orthonormal and complete relations

$$
\left\langle\xi^{\prime} \mid \xi\right\rangle=\frac{\pi}{\sqrt{1-\theta^{2}}} \delta^{(2)}\left(\xi-\xi^{\prime}\right), \quad \frac{\sqrt{1-\theta^{2}}}{\pi} \int d^{2} \xi|\xi\rangle\langle\xi|=1, \quad\left(d^{2} \xi=d \xi_{1} d \xi_{2}\right) .
$$

Thus the eigenstates $|\lambda\rangle$ and $|\xi\rangle$ form two representations for quantum mechanics on the noncommutative phase space, respectively. Since when $\theta=0$ the states $|\lambda\rangle$ and $|\xi\rangle$ are called the continuum entangled states, so one may name these representations in the noncommutative phase space as the entangled state representations. Sometimes working in the $|\lambda\rangle$ or $|\xi\rangle$ representation is convenient, so we first need to know the scalar product of $|\lambda\rangle$ and $|\xi\rangle$. To do this, considering $[\hat{a}, \hat{b}]=0$, we introduce simultaneous eigenstates of $\hat{a}$ and $\hat{b}$, i.e., the two-mode coherent states on the noncommutative phase space

$$
|\alpha, \beta\rangle=\exp \left(\alpha \hat{a}^{\dagger}+\beta \hat{b}^{\dagger}-\alpha^{*} \hat{a}-\beta^{*} \hat{b}\right)|00\rangle,
$$

which satisfies $\hat{a}|\alpha, \beta\rangle=(\alpha+i \theta \beta)|\alpha, \beta\rangle$ and $\hat{b}|\alpha, \beta\rangle=(\beta-i \theta \alpha)|\alpha, \beta\rangle$, respectively. The inner product of two such coherent states is easily to get

$$
\begin{aligned}
\left\langle\alpha^{\prime}, \beta^{\prime} \mid \alpha, \beta\right\rangle= & \exp \left\{-\frac{1}{2}\left(|\alpha|^{2}+|\beta|^{2}+\left|\alpha^{\prime}\right|^{2}+\left|\beta^{\prime}\right|^{2}\right)+\alpha^{\prime *} \alpha+\beta^{* *} \beta\right. \\
& \left.+\frac{i \theta}{2}\left(\beta^{*} \alpha-\alpha^{*} \beta+\beta^{\prime *} \alpha^{\prime}-\alpha^{\prime *} \beta^{\prime}\right)+i \theta\left(\alpha^{\prime *} \beta-\beta^{\prime *} \alpha\right)\right\},
\end{aligned}
$$

which means that the two-mode coherent states are normalized but not orthogonal to each other, and besides, they are over-complete. The corresponding resolution of the identity in the noncommutative phase space is

$$
\left(1-\theta^{2}\right) \int \frac{d^{2} \alpha d^{2} \beta}{\pi^{2}}|\alpha, \beta\rangle\langle\alpha, \beta|=1
$$

where $d^{2} \alpha=d \operatorname{Re} \alpha d \operatorname{Im} \alpha$ and $d^{2} \beta=d \operatorname{Re} \beta d \operatorname{Im} \beta$. Using Eq.(20) one gets

$$
\begin{aligned}
\langle\lambda \mid \xi\rangle & =\frac{1-\theta^{2}}{\pi^{2}} \int d^{2} \alpha d^{2} \beta\langle\lambda \mid \alpha, \beta\rangle\langle\alpha, \beta \mid \xi\rangle \\
& =\frac{1}{2} \exp \left(i\left(\lambda_{1} \xi_{2}-\lambda_{2} \xi_{1}\right)+i \theta\left(\lambda_{1} \xi_{1}-\lambda_{2} \xi_{2}\right)\right) .
\end{aligned}
$$

Having the Eq.(21), one easily gets all of matrix elements of the basic operators $\hat{x}, \hat{y}, \hat{p}_{x}$ and $\hat{p}_{y}$ on the noncommutative phase space in the entangled state representation $|\lambda\rangle$. To 
do this, we only need to evaluate $\left\langle\lambda|\hat{Q}| \lambda^{\prime}\right\rangle$ and $\left\langle\lambda|\hat{K}| \lambda^{\prime}\right\rangle$. With the aid of Eqs.(16) and (17), one gets

$$
\begin{aligned}
\left\langle\lambda|\hat{Q}| \lambda^{\prime}\right\rangle & =\frac{\sqrt{1-\theta^{2}}}{\pi} \int d^{2} \xi\langle\lambda|\hat{Q}| \xi\rangle\left\langle\xi \mid \lambda^{\prime}\right\rangle=i \sqrt{\hbar} \sqrt[4]{\frac{\mu}{\nu}} \frac{\partial}{\partial \lambda_{2}} \delta^{(2)}\left(\lambda-\lambda^{\prime}\right), \\
\left\langle\lambda|\hat{K}| \lambda^{\prime}\right\rangle & =\frac{\sqrt{1-\theta^{2}}}{\pi} \int d^{2} \xi\langle\lambda|\hat{K}| \xi\rangle\left\langle\xi \mid \lambda^{\prime}\right\rangle=-i \sqrt{\hbar} \sqrt[4]{\frac{\nu}{\mu}} \frac{\partial}{\partial \lambda_{1}} \delta^{(2)}\left(\lambda-\lambda^{\prime}\right) .
\end{aligned}
$$

Thus in the $|\lambda\rangle$ representation, we have

$$
\begin{aligned}
\left\langle\lambda|\hat{x}| \lambda^{\prime}\right\rangle & =\sqrt{\frac{\hbar}{2}} \sqrt[4]{\frac{\mu}{\nu}}\left(\lambda_{1}-\theta \lambda_{2}+i \frac{\partial}{\partial \lambda_{2}}\right) \delta^{(2)}\left(\lambda-\lambda^{\prime}\right), \\
\left\langle\lambda|\hat{y}| \lambda^{\prime}\right\rangle & =\sqrt{\frac{\hbar}{2}} \sqrt[4]{\frac{\mu}{\nu}}\left(-\lambda_{1}+\theta \lambda_{2}+i \frac{\partial}{\partial \lambda_{2}}\right) \delta^{(2)}\left(\lambda-\lambda^{\prime}\right), \\
\left\langle\lambda\left|\hat{p}_{x}\right| \lambda^{\prime}\right\rangle & =\sqrt{\frac{\hbar}{2}} \sqrt[4]{\frac{\nu}{\mu}}\left(\lambda_{2}-\theta \lambda_{1}-i \frac{\partial}{\partial \lambda_{1}}\right) \delta^{(2)}\left(\lambda-\lambda^{\prime}\right), \\
\left\langle\lambda\left|\hat{p}_{y}\right| \lambda^{\prime}\right\rangle & =\sqrt{\frac{\hbar}{2}} \sqrt[4]{\frac{\nu}{\mu}}\left(\lambda_{2}-\theta \lambda_{1}+i \frac{\partial}{\partial \lambda_{1}}\right) \delta^{(2)}\left(\lambda-\lambda^{\prime}\right) .
\end{aligned}
$$

Similarly, in the $|\xi\rangle$ representation, we have

$$
\begin{aligned}
\left\langle\xi|\hat{x}| \xi^{\prime}\right\rangle & =\sqrt{\frac{\hbar}{2}} \sqrt[4]{\frac{\mu}{\nu}}\left(\xi_{1}+\theta \xi_{2}+i \frac{\partial}{\partial \xi_{2}}\right) \delta^{(2)}\left(\xi-\xi^{\prime}\right), \\
\left\langle\xi|\hat{y}| \xi^{\prime}\right\rangle & =\sqrt{\frac{\hbar}{2}} \sqrt[4]{\frac{\mu}{\nu}}\left(\xi_{1}+\theta \xi_{2}-i \frac{\partial}{\partial \xi_{2}}\right) \delta^{(2)}\left(\xi-\xi^{\prime}\right), \\
\left\langle\xi\left|\hat{p}_{x}\right| \xi^{\prime}\right\rangle & =\sqrt{\frac{\hbar}{2}} \sqrt[4]{\frac{\nu}{\mu}}\left(\xi_{2}+\theta \xi_{1}-i \frac{\partial}{\partial \xi_{1}}\right) \delta^{(2)}\left(\xi-\xi^{\prime}\right), \\
\left\langle\xi\left|\hat{p}_{y}\right| \xi^{\prime}\right\rangle & =\sqrt{\frac{\hbar}{2}} \sqrt[4]{\frac{\mu}{\nu}}\left(-\xi_{2}-\theta \xi_{1}-i \frac{\partial}{\partial \xi_{1}}\right) \delta^{(2)}\left(\xi-\xi^{\prime}\right) .
\end{aligned}
$$

\section{Wigner operator in the continuum entangled representa- tion}

The so-called Wigner operator (or Wigner-Weyl quantizer) $\hat{\triangle}(x, p)$ is an integral kernel in phase space which transfers a classical function $h(x, p)$ to a quantum operator $\hat{H}(\hat{x}, \hat{p})$

$$
\hat{H}(\hat{x}, \hat{p})=\int d x d p h(x, p) \hat{\triangle}(x, p)
$$

and this quantization prescription is referred to as Weyl correspondence [13]. In fact, because of noncommutativity between different operators in quantum mechanics, the corresponding quantum operator of a classical function is uncertainty. According to Weyl and Wigner [14], the $\hat{\triangle}(x, p)$ is

$$
\hat{\triangle}(x, p)=\frac{1}{(2 \pi \hbar)^{2}} \int d u d v \exp \left(\frac{i}{\hbar} u(\hat{p}-p)+\frac{i}{\hbar} v(\hat{x}-x)\right) .
$$


It is well-known that in ordinary quantum mechanics, in coordinate representation, the Wigner operator $\hat{\triangle}(x, p)$ has the form

$$
\hat{\triangle}(x, p)=\frac{1}{2 \pi} \int d v e^{-i v p}\left|x-\frac{\hbar v}{2}\right\rangle\left\langle x+\frac{\hbar v}{2}\right|,
$$

where $|x\rangle$ is the eigenstate of coordinate operator. Eq.(27) leads to

$$
\operatorname{Tr}\left[\triangle(x, p) \triangle\left(x^{\prime}, p^{\prime}\right)\right]=\frac{1}{2 \pi \hbar} \delta\left(x-x^{\prime}\right) \delta\left(p-p^{\prime}\right) .
$$

Therefore, for a given operator $\hat{H}(\hat{x}, \hat{p})$, its classical correspondence is

$$
h(x, p)=2 \pi \hbar \operatorname{Tr}[\hat{\triangle}(x, p) \hat{H}(\hat{x}, \hat{p})]
$$

and for arbitrary state $|\psi\rangle$, the corresponding Wigner function is $W(x, p)=\langle\psi|\hat{\triangle}(x, p)| \psi\rangle$.

In the noncommutative phase space, the Wigner operator is

$$
\hat{\triangle}(\mathbf{x}, \mathbf{p})=\frac{1}{(2 \pi \hbar)^{4}} \int d \mathbf{u} d \mathbf{v} \exp \left(\frac{i}{\hbar} \mathbf{u} \cdot(\hat{\mathbf{p}}-\mathbf{p})+\frac{i}{\hbar} \mathbf{v} \cdot(\hat{\mathbf{x}}-\mathbf{x})\right),
$$

where $\mathbf{u}=\left(u_{1}, u_{2}\right), \mathbf{v}=\left(v_{1}, v_{2}\right), \mathbf{x}=(x, y), \mathbf{p}=\left(p_{x}, p_{y}\right)$ and $\hat{\mathbf{x}}, \hat{\mathbf{p}}$ are the corresponding quantum operators respectively. Now we consider the WF of the entangled state $|\lambda\rangle$

$$
W_{\lambda}(\mathbf{x}, \mathbf{p})=\frac{1}{(2 \pi \hbar)^{4}} \int d \mathbf{u} d \mathbf{v}\left\langle\lambda\left|\exp \left(\frac{i}{\hbar} \mathbf{u} \cdot(\hat{\mathbf{p}}-\mathbf{p})+\frac{i}{\hbar} \mathbf{v} \cdot(\hat{\mathbf{x}}-\mathbf{x})\right)\right| \lambda\right\rangle .
$$

Since the operators $\hat{x}, \hat{y}, \hat{p}_{x}$ and $\hat{p}_{y}$ obey the commutation relations in Eq.(11), with the aid of the Baker-Campbell-Hausdorff relation, one can write

$$
\begin{aligned}
& \left\langle\lambda\left|\exp \frac{i}{\hbar}\left(u_{1} \hat{p}_{x}+u_{2} \hat{p}_{y}+v_{1} \hat{x}+v_{2} \hat{y}\right)\right| \lambda\right\rangle \\
& =\quad \exp \left(\frac{i}{2 \hbar^{2}}\left(\mu v_{1} v_{2}+\nu u_{1} u_{2}\right)+\frac{i}{2 \hbar}\left(u_{1} v_{1}+u_{2} v_{2}\right)\right) \\
& \times\left\langle\lambda\left|\exp \left(\frac{i}{\hbar} v_{1} \hat{x}\right) \exp \left(\frac{i}{\hbar} u_{1} \hat{p}_{x}\right) \exp \left(\frac{i}{\hbar} v_{2} \hat{y}\right) \exp \left(\frac{i}{\hbar} u_{2} \hat{p}_{y}\right)\right| \lambda\right\rangle .
\end{aligned}
$$

Employing Eq.(23) it is not difficult to evaluate the above matrix element

$$
\begin{aligned}
\left\langle\lambda\left|\exp \left(\frac{i}{\hbar} v_{1} \hat{x}\right) \exp \left(\frac{i}{\hbar} u_{1} \hat{p}_{x}\right) \exp \left(\frac{i}{\hbar} v_{2} \hat{y}\right) \exp \left(\frac{i}{\hbar} u_{2} \hat{p}_{y}\right)\right| \lambda\right\rangle=2 \pi \hbar \delta\left(u_{1}-u_{2}\right) \delta\left(v_{1}+v_{2}\right) \\
\times \exp \left(\frac{i}{4 \hbar^{2}}\left(\mu\left(v_{1}^{2}-2 v_{1} v_{2}-v_{2}^{2}\right)-\nu\left(u_{1}^{2}+2 u_{1} u_{2}-u_{2}^{2}\right)\right)-\frac{i}{2 \hbar}\left(u_{1}+u_{2}\right)\left(v_{1}+v_{2}\right)\right) \\
\times \exp \left(\frac{i}{\sqrt{2 \hbar}}\left(\sqrt[4]{\frac{\mu}{\nu}}\left(v_{1}-v_{2}\right)\left(\lambda_{1}-\theta \lambda_{2}\right)+\sqrt[4]{\frac{\nu}{\mu}}\left(u_{1}+u_{2}\right)\left(\lambda_{2}-\theta \lambda_{1}\right)\right)\right) .
\end{aligned}
$$

Substituting these results into Eq.(31), after integrating over the variables $\mathbf{u}$ and $\mathbf{v}$, one gets

$$
W_{\lambda}(\mathbf{x}, \mathbf{p})=\frac{1}{2 \pi \hbar \sqrt{1-\theta^{2}}} \delta\left(x-y-\sqrt{2 \hbar} \sqrt[4]{\frac{\mu}{\nu}}\left(\lambda_{1}-\theta \lambda_{2}\right)\right) \delta\left(p_{x}+p_{y}-\sqrt{2 \hbar} \sqrt[4]{\frac{\nu}{\mu}}\left(\lambda_{2}-\theta \lambda_{1}\right)\right),
$$

which is consistent with the eigenvalue equations in Eq.(8). 
Furthermore, one can get an explicit form of $\hat{\triangle}(\mathbf{x}, \mathbf{p})$ in the entangled representation $|\lambda\rangle$. Using Eq.(12) one has

$$
\hat{\triangle}(\mathbf{x}, \mathbf{p})=\frac{1-\theta^{2}}{\pi^{2}(2 \pi \hbar)^{4}} \int d \mathbf{u} d \mathbf{v} d^{2} \lambda d^{2} \lambda^{\prime}|\lambda\rangle\left\langle\lambda\left|\exp \left(\frac{i}{\hbar} \mathbf{u} \cdot(\hat{\mathbf{p}}-\mathbf{p})+\frac{i}{\hbar} \mathbf{v} \cdot(\hat{\mathbf{x}}-\mathbf{x})\right)\right| \lambda^{\prime}\right\rangle\left\langle\lambda^{\prime}\right| .
$$

After integrating over the variables $\mathbf{u}$ and $\mathbf{v}$, one gets

$$
\begin{aligned}
\hat{\triangle}(\mathbf{x}, \mathbf{p}) & =\frac{\sqrt{1-\theta^{2}}}{2 \pi^{3} \hbar} \int d^{2} \lambda d^{2} \lambda^{\prime} \delta\left(\lambda_{1}+\lambda_{1}^{\prime}-\theta\left(\lambda_{2}+\lambda_{2}^{\prime}\right)-\sqrt{\frac{2}{\hbar}} \sqrt[4]{\frac{\nu}{\mu}}(x-y)\right) \\
& \times \quad \delta\left(\lambda_{2}+\lambda_{2}^{\prime}-\theta\left(\lambda_{1}+\lambda_{1}^{\prime}\right)-\sqrt{\frac{2}{\hbar}} \sqrt[4]{\frac{\mu}{\nu}}\left(p_{x}+p_{y}\right)\right) \\
& \times \exp \left(i\left(\lambda_{1} \lambda_{2}^{\prime}-\lambda_{2} \lambda_{1}^{\prime}\right)+i \frac{\theta}{2}\left(\lambda_{1}^{\prime 2}-\lambda_{2}^{\prime 2}-\lambda_{1}^{2}+\lambda_{2}^{2}\right)\right) \\
& \times \exp \left(-i \sqrt{\frac{2}{\hbar}}\left(\sqrt[4]{\frac{\mu}{\nu}} p_{y}\left(\lambda_{1}-\lambda_{1}^{\prime}\right)+\sqrt[4]{\frac{\nu}{\mu}} y\left(\lambda_{2}-\lambda_{2}^{\prime}\right)\right)\right)|\lambda\rangle\left\langle\lambda^{\prime}\right| .
\end{aligned}
$$

Then performing the integration over $\lambda$ and introducing the following notations

$$
\begin{array}{ll}
\frac{1}{\sqrt{2 \hbar}\left(1-\theta^{2}\right)} \sqrt[4]{\frac{\nu}{\mu}}(x-y)=\rho_{1}, & \frac{1}{\sqrt{2 \hbar}\left(1-\theta^{2}\right)} \sqrt[4]{\frac{\mu}{\nu}}\left(p_{x}+p_{y}\right)=\rho_{2}, \\
\frac{1}{\sqrt{2 \hbar}\left(1-\theta^{2}\right)} \sqrt[4]{\frac{\nu}{\mu}}(x+y)=\gamma_{1}, & \frac{1}{\sqrt{2 \hbar}\left(1-\theta^{2}\right)} \sqrt[4]{\frac{\mu}{\nu}}\left(p_{x}-p_{y}\right)=\gamma_{2},
\end{array}
$$

one can write the above expression as

$$
\begin{aligned}
& \hat{\triangle}(\mathbf{x}, \mathbf{p})= \\
& =\frac{\sqrt{1-\theta^{2}}}{\pi^{3} \hbar^{2}} \int d^{2} \lambda^{\prime} \exp \left(-2 i\left(\left(\rho_{2}-\theta \rho_{1}\right)\left(\lambda_{1}^{\prime}-\theta \lambda_{2}^{\prime}\right)-\left(\rho_{1}-\theta \rho_{2}\right)\left(\lambda_{2}^{\prime}-\theta \lambda_{1}^{\prime}\right)\right)\right) \\
& \quad \times \exp \left(2 i\left(1-\theta^{2}\right)\left(\left(\rho_{2}-\gamma_{2}\right) \lambda_{1}^{\prime}-\left(\rho_{1}-\gamma_{1}\right) \lambda_{2}^{\prime}-\gamma_{1}\left(\rho_{2}+\theta \rho_{1}\right)+\gamma_{2}\left(\rho_{1}+\theta \rho_{2}\right)\right)\right) \\
& \quad \times\left|2\left(\rho_{1}+\theta \rho_{2}\right)-\lambda_{1}^{\prime}, 2\left(\rho_{2}+\theta \rho_{1}\right)-\lambda_{2}^{\prime}\right\rangle\left\langle\lambda_{1}^{\prime}, \lambda_{2}^{\prime}\right| .
\end{aligned}
$$

At last, in Eq.(38) using new integration variables $\lambda$ to replace the old ones $\lambda^{\prime}$

$$
\rho_{1}+\theta \rho_{2}-\lambda_{1}^{\prime}=\lambda_{1}, \quad \rho_{2}+\theta \rho_{1}-\lambda_{2}^{\prime}=\lambda_{2},
$$

one obtains the expression of the Wigner operator in the entangled state $|\lambda\rangle$ representation

$$
\begin{aligned}
\hat{\triangle}(\mathbf{x}, \mathbf{p}) & =\frac{\sqrt{1-\theta^{2}}}{\pi^{3} \hbar^{2}} \int d^{2} \lambda \exp \left(2 i\left(1-\theta^{2}\right)\left(\gamma_{1} \lambda_{2}-\gamma_{2} \lambda_{1}\right)\right) \\
& \times\left|\rho_{1}+\theta \rho_{2}-\lambda_{1}, \rho_{2}+\theta \rho_{1}-\lambda_{2}\right\rangle\left\langle\rho_{1}+\theta \rho_{2}+\lambda_{1}, \rho_{2}+\theta \rho_{1}+\lambda_{2}\right|,
\end{aligned}
$$

where the ket and bra vector are in the $|\lambda\rangle$ representation. Since the entangle states $|\lambda\rangle$ are orthonormal to each other (see Eq.(11)), it is very easy to get the WF $W_{\lambda}(\mathbf{x}, \mathbf{p})$ (34) from the expression (40). The only needed to do is take the expectation value of the Wigner operator (40) in the state $|\lambda\rangle$.

Similarly, one can get the WF of the entangled state $|\xi\rangle$

$$
W_{\xi}(\mathbf{x}, \mathbf{p})=\frac{1}{2 \pi \hbar \sqrt{1-\theta^{2}}} \delta\left(x+y-\sqrt{2 \hbar} \sqrt[4]{\frac{\mu}{\nu}}\left(\xi_{1}+\theta \xi_{2}\right)\right) \delta\left(p_{x}-p_{y}-\sqrt{2 \hbar} \sqrt[4]{\frac{\nu}{\mu}}\left(\xi_{2}+\theta \xi_{1}\right)\right)
$$


which is consistent with the eigenvalue equations in Eq.(16). Using the inner product (21), from Eq.(40) one has the explicit expression of the Wigner operator in the entangled state $|\xi\rangle$ representation

$$
\begin{aligned}
\hat{\triangle}(\mathbf{x}, \mathbf{p}) & =\frac{\sqrt{1-\theta^{2}}}{\pi^{3} \hbar^{2}} \int d^{2} \xi \exp \left(2 i\left(1-\theta^{2}\right)\left(\rho_{1} \xi_{2}-\rho_{2} \xi_{1}\right)\right) \\
& \times\left|\gamma_{1}-\theta \gamma_{2}-\xi_{1}, \gamma_{2}-\theta \gamma_{1}-\xi_{2}\right\rangle\left\langle\gamma_{1}-\theta \gamma_{2}+\xi_{1}, \gamma_{2}-\theta \gamma_{1}+\xi_{2}\right|
\end{aligned}
$$

where the ket and bra vector are in the $|\xi\rangle$ representation.

\section{Some possible applications}

It is well know that representation plays a basic role in quantum mechanics like the coordinate systems in geometry. In section 2 we introduced the entangled state representations $|\lambda\rangle$ and $|\xi\rangle$. In the $|\lambda\rangle$ or $|\xi\rangle$ representation one can also solve problems of NCQM, and sometimes it is more convenient working in the entangled state representation than in other representation. To show this, let us study a two-dimensional harmonic oscillator on the noncommutative phase space with both momentum-momentum (kinetic) coupling and coordinate-coordinate (elastic) coupling. The quantum Hamiltonian is

$$
\hat{H}=\frac{1}{2 m}\left(\hat{p}_{x}^{2}+\hat{p}_{y}^{2}\right)+\frac{m \omega^{2}}{2}\left(\hat{x}^{2}+\hat{y}^{2}\right)+\frac{k}{2}(\hat{x} \hat{y}+\hat{y} \hat{x})+\frac{l}{2}\left(\hat{p}_{x} \hat{p}_{y}+\hat{p}_{y} \hat{p}_{x}\right),
$$

where the operators $\hat{p}_{x}, \hat{p}_{y}, \hat{x}$ and $\hat{y}$ satisfy the commutation relations (11). If one use the usual Seiberg-Witten map to rewrite this Hamiltonian in terms of some kind of commutative coordinate and momentum operators, one will have a very complicated expression, which includes not only the kinetic and the elastic coupling terms, but also the coordinatemomentum coupling terms (they are the angular momentum term and the squeezing term, respectively). It is not an easy task to solve its energy spectra. However, in the $|\lambda\rangle$ representation the Hamiltonian $\hat{H}$ (43) has a very simple form

$$
\hat{H}=c_{1} \eta_{1}^{2}+c_{2} \eta_{2}^{2}+d_{1} \lambda_{1}^{2}+d_{2} \lambda_{2}^{2}+d_{3} \lambda_{1} \lambda_{2},
$$

where $\eta_{1}=-i \partial / \partial \lambda_{1}, \eta_{2}=-i \partial / \partial \lambda_{2}$, which satisfy the standard Heisenberg commutation relations

$$
\left[\lambda_{i}, \eta_{j}\right]=i \delta_{i j}, \quad\left[\lambda_{1}, \lambda_{2}\right]=\left[\eta_{1}, \eta_{2}\right]=0 .
$$

In Eq.(44), the coefficients $c_{1}, c_{2}, d_{1}, d_{2}, d_{3}$ are

$$
\begin{aligned}
c_{1} & =\frac{\hbar}{2 m} \sqrt{\frac{\nu}{\mu}}(1-l m), \quad c_{2}=\frac{\hbar m \omega^{2}}{2} \sqrt{\frac{\mu}{\nu}}\left(1+\frac{k}{m \omega^{2}}\right), \\
d_{1} & =\frac{\hbar \theta^{2}}{2 m} \sqrt{\frac{\nu}{\mu}}(1+l m)+\frac{\hbar m \omega^{2}}{2} \sqrt{\frac{\mu}{\nu}}\left(1-\frac{k}{m \omega^{2}}\right), \\
d_{2} & =\frac{\hbar}{2 m} \sqrt{\frac{\nu}{\mu}}(1+l m)+\frac{\hbar \theta^{2} m \omega^{2}}{2} \sqrt{\frac{\mu}{\nu}}\left(1-\frac{k}{m \omega^{2}}\right), \\
d_{3} & =-\frac{\nu}{m}(1+l m)-\mu m \omega^{2}\left(1-\frac{k}{m \omega^{2}}\right),
\end{aligned}
$$


respectively. Thus the two-dimensional harmonic oscillator on the noncommutative phase space with both momentum-momentum (kinetic) coupling and coordinate-coordinate (elastic) coupling is transferred to a very simple ordinary two-dimensional coupling oscillator in the $|\lambda\rangle$ representation, and the corresponding energy spectra and WFs are easily to get.

In fact, one can make a scaling change and a planar rotation to reduce the Hamiltonian (44) further [15, 16. To do this, we make the following scaling changes

$$
\lambda_{1}^{\prime}=\sqrt[4]{\frac{c_{2}}{c_{1}}} \lambda_{1}, \quad \lambda_{2}^{\prime}=\sqrt[4]{\frac{c_{1}}{c_{2}}} \lambda_{2}, \quad \eta_{1}^{\prime}=\sqrt[4]{\frac{c_{1}}{c_{2}}} \eta_{1}, \quad \eta_{2}^{\prime}=\sqrt[4]{\frac{c_{2}}{c_{1}}} \eta_{2},
$$

which lead the Hamiltonian (44) to

$$
\hat{H}=c\left(\eta_{1}^{\prime 2}+\eta_{2}^{\prime 2}\right)+f_{1} \lambda_{1}^{\prime 2}+f_{2} \lambda_{2}^{\prime 2}+d_{3} \lambda_{1}^{\prime} \lambda_{2}^{\prime},
$$

where

$$
\begin{aligned}
c & =\sqrt{c_{1} c_{2}}=\frac{\hbar \omega}{2} \sqrt{(1-l m)\left(1+\frac{k}{m \omega^{2}}\right)} \\
f_{1} & =d_{1} \sqrt{\frac{c_{1}}{c_{2}}}=\left(\frac{\hbar \omega}{2}\left(1-\frac{k}{m \omega^{2}}\right)+\frac{\nu^{2}}{2 m^{2} \hbar \omega}(1+l m)\right) \sqrt{\frac{1-l m}{1+\frac{k}{m \omega^{2}}}} \\
f_{2} & =d_{2} \sqrt{\frac{c_{2}}{c_{1}}}=\left(\frac{\hbar \omega}{2}(1+l m)+\frac{\mu^{2} m^{2} \omega^{3}}{2 \hbar}\left(1-\frac{k}{m \omega^{2}}\right)\right) \sqrt{\frac{1+\frac{k}{m \omega^{2}}}{1-l m}} .
\end{aligned}
$$

Then, since the Hamiltonian (48) still involves an interaction term, we can simplify this situation by a transformation to new phase space variables

$$
x_{i}=u_{i j} \lambda_{j}^{\prime}, \quad p_{i}=u_{i j} \eta_{j}^{\prime}, \quad(i, j=1,2)
$$

where the matrix

$$
\left(u_{i j}\right)=\left(\begin{array}{cc}
\cos \frac{\alpha}{2} & -\sin \frac{\alpha}{2} \\
\sin \frac{\alpha}{2} & \cos \frac{\alpha}{2}
\end{array}\right)
$$

is a unitary rotation with the mixing angle $\alpha$. When $\alpha$ satisfies the condition

$$
\tan \alpha=\frac{d_{3}}{f_{2}-f_{1}},
$$

the Hamiltonian (48) has a factorizing form

$$
\hat{H}=\frac{1}{2 M} p_{1}^{2}+\frac{M \Omega_{+}^{2}}{2} x_{1}^{2}+\frac{1}{2 M} p_{2}^{2}+\frac{M \Omega_{-}^{2}}{2} x_{2}^{2},
$$

where

$$
\begin{aligned}
M & =\left(\hbar \omega \sqrt{(1-\operatorname{lm})\left(1+\frac{k}{m \omega^{2}}\right)}\right)^{-1}, \\
\Omega_{ \pm} & =\hbar \omega\left(\sqrt{A_{+}+B_{-}} \pm \sqrt{A_{-}+B_{+}}\right)
\end{aligned}
$$


where

$$
\begin{aligned}
& A_{ \pm}=\frac{1}{2}\left(1+\frac{k l}{\omega^{2}} \pm \sqrt{\left(1-l^{2} m^{2}\right)\left(1-\frac{k^{2}}{m^{2} \omega^{4}}\right)}\right) \\
& B_{ \pm}=\left(\frac{\nu \sqrt{1-l^{2} m^{2}}}{2 m \hbar \omega} \pm \frac{\mu m \omega}{2 \hbar} \sqrt{1-\frac{k^{2}}{m^{2} \omega^{4}}}\right)^{2} .
\end{aligned}
$$

Obviously, the transformations (47) and (50) do not change the standard Heisenberg commutation relations, so the Hamiltonian (53) describes a two-dimensional uncoupling harmonic oscillator and its energy level as well as WFs are well-known

$$
E_{n_{1} n_{2}}=\left(n_{1}+\frac{1}{2}\right) \Omega_{+}+\left(n_{2}+\frac{1}{2}\right) \Omega_{-}
$$

and

$$
W_{n_{1} n_{2}}=4(-1)^{n_{1}+n_{2}} e^{-2 H_{1} / \Omega_{+}} e^{-2 H_{2} / \Omega_{-}} L_{n_{1}}\left(\frac{4 H_{1}}{\Omega_{+}}\right) L_{n_{2}}\left(\frac{4 H_{2}}{\Omega_{-}}\right)
$$

respectively, where $L_{n}$ are the Laguerre polynomials and $H_{1}=\frac{1}{2 M} p_{1}^{2}+\frac{M \Omega_{+}^{2}}{2} x_{1}^{2}, H_{2}=$ $\frac{1}{2 M} p_{2}^{2}+\frac{M \Omega_{-}^{2}}{2} x_{2}^{2}$, and here $x_{1}, x_{2}$ and $p_{1}, p_{2}$ are classical phase space variables.

The energy (55) will reduce to some familiar results in several specific situations, for instance, when $\mu, \nu=0$, i.e., in the ordinary commutative phase space, (55) becomes

$$
\begin{aligned}
E_{n_{1} n_{2}}= & \left(n_{1}+n_{2}+1\right) \frac{\hbar \omega}{\sqrt{2}} \sqrt{1+\frac{k l}{\omega^{2}}+\sqrt{\left(1-l^{2} m^{2}\right)\left(1-\frac{k^{2}}{m^{2} \omega^{4}}\right)}} \\
& +\left(n_{1}-n_{2}\right) \frac{\hbar \omega}{\sqrt{2}} \sqrt{1+\frac{k l}{\omega^{2}}-\sqrt{\left(1-l^{2} m^{2}\right)\left(1-\frac{k^{2}}{m^{2} \omega^{4}}\right)}}
\end{aligned}
$$

when $k, l=0$ and $\mu, \nu \neq 0$, (55) becomes

$$
E_{n_{1} n_{2}}=\left(n_{1}+n_{2}+1\right) \hbar \omega \sqrt{1+\frac{\left(\nu-\mu m^{2} \omega^{2}\right)^{2}}{4 m^{2} \hbar^{2} \omega^{2}}}+\left(n_{1}-n_{2}\right)\left(\frac{\nu}{2 m}+\frac{\mu m \omega^{2}}{2}\right) ;
$$

and when both $\mu, \nu=0$ and $k, l=0$, the energy has familiar form $E_{n_{1} n_{2}}=\left(n_{1}+n_{2}+1\right) \hbar \omega$.

\section{Summary and discussion}

In order to develop representation and transformation theory so that one can solve more dynamic problems for NCQM, employing the EPR idea we construct simultaneous eigenstates of the difference (or the sum) of two different coordinate-component operators and the sum (or the difference) of two relevant momentum operators. Since these new state-vectors are orthonormal and satisfy the completeness relation, they form representations to formulate the NCQM and we name them the entangled state representations. We also derive explicit expressions of Wigner operator and WFs in the new representations. In order to show the potential role of the new representations in NCQM, we study a two-dimensional oscillator with both kinetic and elastic couplings on the noncommutative phase space and simply get its exact energy spectra and WFs in the entangled state representation. 
It is worth pointing out that the new entangled state representations are built based on the deformed boson algebra (44). Also form the deformed boson algebra (4), one can construct the coherent state representation and the squeezed state representation on the noncommutative phase space. Thus although there are no the standard coordinate and the standard momentum representations on the noncommutative phase space, one can develop the coherent state, the squeezed state and the entangled state and other useful representations to solve physics problems on the noncommutative phase space. We would like also to emphasize that, in contrast to the most work on the NCQM in the literature, our work is done directly in the noncommutative phase space without using any variables in the ordinary commutative phase space. So if one thinks the usual way to NCQM (transferring problems in the noncommutative space into corresponding ones in the commutative space) as the "perturbation approach", our way may be named as "nonperturbation approach".

It is also interesting to develop the theory of representations and transformations on the noncommutative phase space further and work on this direction will be presented in a separate paper.

\section{Acknowledgments}

This project was supported by the National Natural Science Foundation of China under Grant 10675106.

\section{References}

[1] P. A. M. Dirac, The Principles of Quantum Mechanics, Oxford Clarendon Press, 1930.

[2] M. Chaichian, M. M. Sheikh-Jabbari and A. Tureanu, Phys. Rev. Lett. 86 (2001) 2716.

[3] J. Gamboa, M. Loewe and J. C. Rojas, Phys. Rev. D 64 (2001) 067901.

[4] X. Calmet and M. Selvaggi, Phys. Rev. D 74 (2006) 037901.

[5] O. Bertolami, et al, Phys. Rev. D 72 (2005) 025010.

[6] M. Rosenbaum and J. D. Vergara, Gen. Relativ. Gravit. 38 (2006) 607.

[7] T. P. Singh, S. Gutti and R. Tibrewala, gr-qc/0503116.

[8] A. Einstein, B. Podolsky and N. Rosen, Phys. Rev. 47 (1935) 777.

[9] H. Fan and J. R. Klauder, Phys. Rev. A 49 (1994) 704.

[10] J. Zhang, Phys. Rev. Lett. 93 (2004) 043002, hep-th/0405143.

[11] C. M. Caves and B. L. Schumaker, Phys. Rev. A 31, (1985) 3068; B. L. Schumaker, C. M. Caves, Phys. Rev. A 31, (1985) 3093.

[12] O.Bertolami and J. G. Rosa, Mod. Phys. Lett. A 21 (2006) 795.

[13] H. Weyl, Zeitsch. Phys. 46 (1927) 1.

[14] E. Wigner, Phys. Rev. 40 (1932) 749. 
[15] D. Han, Y. Kim and M. Noz, J. Math. Phys. 36 (1995) 3940.

[16] A. Jellal, et al, Int. J. Mod. Phys. A 20 (2005) 7. 